\title{
EMPLEO DE LA NINTENDO WII PARA LA MEJORA DEL RENDIMIENTO ACADÉMICO
}

\author{
Sebastián López Serrano \\ Departamento de Didáctica de la Expresión Musical, Plástica y Corporal. \\ Facultad de Humanidades y Ciencias de la Educación. Universidad de Jaén. \\ Email: sls00013@red.ujaen.es
}

https://doi.org/10.17060/ijodaep.2017.n1.v3.1017

Fecha de Recepción: 1 Marzo 2017

Fecha de Admisión: 1 Abril 2017

\section{RESUMEN}

Actualmente nos encontramos ante una sociedad en constante evolución en busca de nuevas estrategias metodológicas que permitan un mejor desarrollo integral de la persona. Estas deben ser atractivas y motivadoras a la vez que permitan contribuir a mejorar las competencias sociales y cognitivas. Dentro del ámbito educativo, nos encontramos ante una situación compleja puesto que los últimos informes arrojados a nivel europeo ponen de relieve un bajo nivel en cuanto a rendimiento académico.

La evidencia científica actual ha corroborado que una manera de estimular la función cognitiva es a través de la práctica habitual de Actividad Física (AF) (Ruiz-Ariza et al., 2017a). Se ha demostrado que con la inclusión de actividades lúdicas como los Video Juegos Activos durante la jornada académica ayuda a incrementar la cantidad de AF practicada, así como a mejorar la socialización y el rendimiento cognitivo 3/4atención, concentración, memoria3\%4 (Joronen et al., 2016).

Este trabajo pretende poner de manifiesto como la práctica de AF mediante el uso de la videoconsola Nintendo Wii influye de manera positiva en la cognición de niños y adolescentes. Para ello realizaremos una búsqueda bibliográfica en los últimos 7 años. Los resultados obtenidos muestran una asociación de la práctica de la Nintendo Wii con una mejora en la cognición e incremento del nivel de AF. Como conclusión, se sugiere el empleo de las nuevas tecnologías, sobre todo, usándolas de forma integral mediante juegos educativos que conlleven movimiento de forma implícita, para continuar inculcando hábitos saludables de actividad física, aumentar la motivación del alumnado, reducir el tiempo sedentario diario que pasan en las aulas, favorecer a una mejor socialización, y consecuentemente, mejorar el rendimiento escolar.

\section{Palabras Clave}

Nintendo Wii, exergames, actividad física, cognición, rendimiento académico. 


\title{
EMPLEO DE LA NINTENDO WII PARA LA MEJORA DEL RENDIMIENTO ACADÉMICO
}

\begin{abstract}
Use of the nintendo wii for the improvement of academic performance

Nowadays, we live in a society in constant evolution in search of new methodological strategies that allow to achieve a better integral development of the person. These should be attractive and motivational, while contributing to the improvement of social and cognitive skills. Within the educational field, we are faced with a complex situation since the latest reports at European level highlight a low level of academic performance.

Currently scientific evidence has corroborated that one way of stimulating cognitive function is through the usual practice of Physical Activity (PA) (Ruiz-Ariza et al., 2017). It has been shown that with the inclusion of play activities such as Active Video Games during the school days helps to increase the amount of practiced PA, as well as to improve socialization and cognitive performance $3 / 4$ attention, concentration, memory $3 / 4$ (Joronen et al., 2016).

This work aims to show the practice of PA through the use of the Nintendo Wii console have positively influences about cognition of children and adolescents. For this, we will carry out a bibliographic search in the last 7 years. The results show an association of the practice of Nintendo Wii with an improvement in cognition and increase of the level of PA. In conclusion, the use of new technologies is suggested, above all, using them in an integral way through educational games that involve movement implicitly, to continue instilling healthy habits of physical activity, to increase student's motivation, to reduce the daily sedentary time spend in classrooms, favour a better socialization and consequently, improve school performance.
\end{abstract}

\section{Keywords}

Nintendo Wii, exergames, physical activity, cognition, academic performace.

\section{ANTECEDENTES}

Recientes estudios han demostrado que la práctica sistemática de Actividad Física (AF) puede contribuir a la mejora del Rendimiento Cognitivo (RC) en niños (Happala, 2013) y adolescentes (Ardoy et al., 2014). La AF produce adaptaciones fisiológicas como el aumento del flujo sanguíneo aumentando los niveles del factor neurotrófico derivado del cerebro (BDNF) que promueve el crecimiento y supervivencia de las neuronas, mejorando la capacidad de aprendizaje (Chaddock et al., 2014). Además, la práctica de AF ayuda a reducir los altos niveles de sedentarismo que nos encontramos actualmente siendo un gran recurso para la mejora de la salud, prevención de enfermedades 0 aumento de la esperanza de vida (OMS, 2015).

En relación a lo anterior, los videojuegos activos como la Nintendo Wii, aparecen como una oportunidad clave para aumentar la cantidad diaria de AF de una forma lúdica. Estos video juegos activo presentan una gran popularidad entre los jóvenes, y su dinámica interactiva genera una conexión entre el jugador y el juego. De esta manera, se interpretan los movimientos corporales reflejando la actividad realizada durante su desarrollo en el dispositivo. Además, este tipo de modalidad de video juego favorece a las relaciones sociales entre compañeros (Nurkkala, Kalermo \& Jarvilehto, 2014) e incrementan el gasto calórico (Dutta \& Pereira, 2015). Por otra parte, se conoce el potencial que los Exergames (EX) presentan en la transformación del tiempo sedentario en tiempo físicamente activo (LeBlanc \& Chaput, 2016). Por lo tanto, conocer la relación del empleo de los EX en el marco educativo podría favorecer a incrementar los niveles de AF, la reducción del tiempo sedentario que actualmente pasan los niños en el aula (Norris et al., 2016) y favorecer a su desarrollo personal y social, con consecuencias directas sobre el rendimiento escolar. 


\section{METOLOGíA}

\section{Objetivo}

Realizar una revisión bibliográfica actual que se asocie el efecto de la Nintendo Wii con el rendimiento académico en niños y adolescentes.

\section{Muestra de Participantes}

La edad de los participantes analizados osciló entre los 6 y 19 años. El total de participantes teniendo en cuenta estas investigaciones fue de 252 niños y adolescentes.

Se usaron dos bases de datos especializadas (Web of Science y SportDiscus) y se limitó la búsqueda a los últimos 7 años. Los términos de búsqueda usados se pueden observar en la tabla 1. La búsqueda se realizó en inglés.

Tabla 1.

Estrategias de búsqueda en las bases de datos.

\begin{tabular}{|c|c|c|c|}
\hline Database & Search Strategy & Limits & Filter \\
\hline Web of Science & $\begin{array}{l}\text { Topic: (exergames } \\
\text { OR Nintendo Wii) } \\
\text { AND Topic: } \\
\text { (cognitive OR } \\
\text { academic outcomes } \\
\text { OR academic } \\
\text { achievement) AND } \\
\text { Topic: (Children } \\
\text { OR adolescent) }\end{array}$ & $\begin{array}{l}\text {-Publication date } \\
\text { from } 2010 / 01 / 01 \text { to } \\
2017 / 01 / 01 \\
\text {-English language } \\
\text {-Article or review }\end{array}$ & 14 items filtered \\
\hline $\begin{array}{l}\text { SportDiscus } \\
\text { (EBSCO) }\end{array}$ & \begin{tabular}{l}
\multicolumn{3}{c}{ TX (Exergames OR } \\
Nintendo Wii) \\
AND \\
(Cognitive OR \\
academic outcomes \\
OR academic \\
achievement) AND \\
TX (chidren OR \\
adolescents)
\end{tabular} & $\begin{array}{l}\text {-Publication date } \\
\text { from } 2010 / 01 / 01 \text { to } \\
2017 / 01 / 01 \\
\text {-English language }\end{array}$ & 112 items filtered \\
\hline
\end{tabular}

Los criterios de selección que se utilizaron fueron:

1. El estudio estuviera realizado con población sana.

2. La población fuera de niños y adolescentes entre 6 y 18 años.

3. El video juego y la variable de rendimiento académico estuvieran claramente descritos.

\section{RESULTADOS}

El flow de los resultados obtenidos tras la realización de la búsqueda se muestra en la Figura 1. Después de la eliminación de los duplicados, excluidos por el título o edad, un total de 10 artículos fueron revisados. Estos estudios siguieron los criterios de revisión expuestos anteriormente. Finalmente, se incluyeron 4 artículos para su análisis. 


\section{EMPLEO DE LA NINTENDO WII PARA LA MEJORA DEL RENDIMIENTO ACADÉMICO}

Figura 1.

Flujo de artículos a través del proceso de búsqueda.

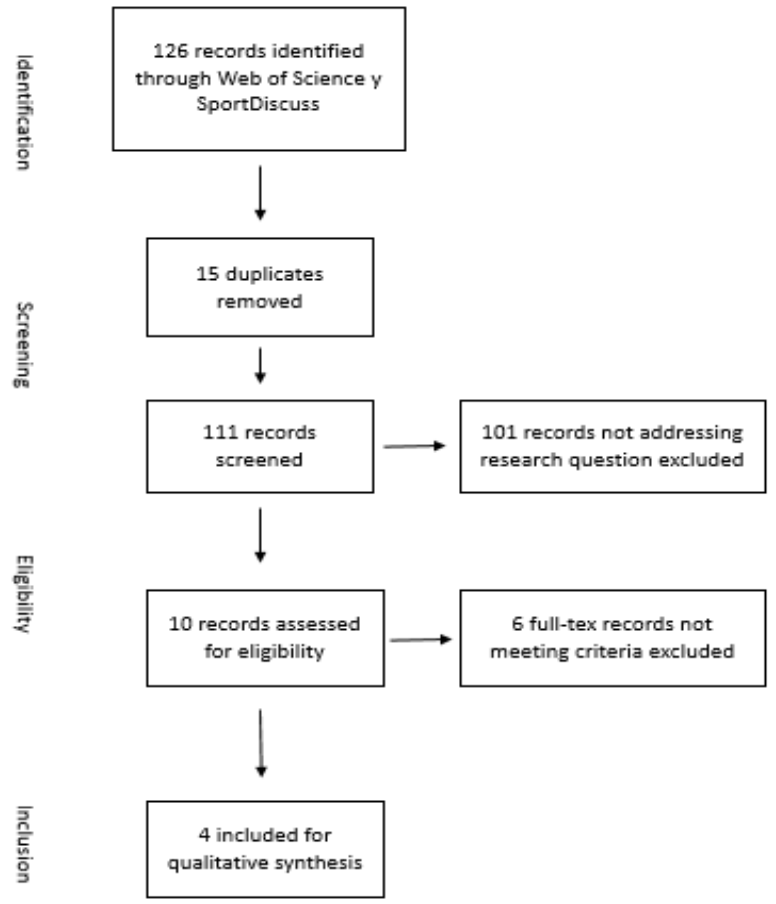

Los cuatro estudios seleccionados fueron estudios de intervención (Best, 2011; Flynn, Richert, Staiano, Wartella \& Calvert, 2014; Gao, Lee, Pope, \& Zhang, 2016; Staiano, Abraham \& Calvert, 2012). 2 estudios realizaron los grupos de manera aleatoria (Flynn et al., 2014; Staiano et al., 2012) y otros 2 no realizaron aleatorización de grupos (Best, 2011; Gao et al., 2016). Presentan un total de 252 sujetos. Todos ellos pertenecen al continente americano (USA). Las variables de AF que se controlaron fueron la frecuencia cardiaca (Best, 2011), resistencia y fuerza (Staiano et al., 2012), la puntuación dada por el juego tras la sesión de práctica (Gao et al., 2016) y el tiempo en recorrer una milla antes y después de la intervención con la Nintendo Wii (Gao et al., 2016). Las medidas de rendimiento a nivel cognitivo se centraron en las funciones ejecutivas $3 / 4$ esfuerzo académico, nivel de logro, frustración, aburrimiento, autoconcepto $3 / 4$. Estos estudios incluyeron covariables, entre las que destacan el nivel socioeducativo de las familias, el IMC, el sexo y la edad. Los juegos utilizados en estos estudios corresponden con versiones específicas para Nintendo Wii, como son "Active Life" 3/4Se utiliza una plataforma para jugar a minijuegos $3 / 4$, "EA Sports Active" 3/4actividades que usan la detección del movimiento de la Wii3/4 y "Nintendo Wii Fit"3/4Se desarrolla en una superficie que evalúa el estado físico mientras se desarrollan diferentes actividades $3 / 4$.

Los estudios mostraron una relación positiva entre el nivel AF realizada durante el EX y la cognición. Best (2011) demostró que con una sesión de EX mejoraron las funciones cognitivas de los niños. Staiano et al. (2012), tras diez semanas de intervención, indicó que los jóvenes que practicaron EX de manera competitiva y cooperativa mejoraron sus funciones ejecutivas más que el grupo 
que no realizó ningún tipo de actividad mediante estos dispositivos. Por su parte, Flynn et al. (2014), demostró que los niños que jugaron durante cinco semanas a EX mejoraron su rendimiento cognitivo, y a medida que más jugaban, obtuvieron mayores beneficios. Gao et al. (2016), tras la realización de una intervención de seis semana concluyó que los niños sometidos a la práctica de EX obtuvieron mejores resultados en relación con las funciones ejecutivas y esfuerzo.

\section{DISCUSIÓN}

Los resultados de esta revisión están relacionados con la mayoría de las investigaciones previas que relacionan una asociación positiva entre el juego de cualquier modalidad de EX y la mejora de la cognición (Joronen, et al., 2016; Norris et al., 2016). Estos estudios mostraron los beneficios en aspectos cognitivos — atención, comprensión y motivación— como de rendimiento académico. Otros estudios, se centraron en los beneficios que los video juegos activos presenta sobre la salud (Gao, Chen, Pasco y Pope, 2015-, Dutta, 2015), influyendo positivamente en un incremento del gasto calórico y mejor nivel de condición física. Por otro lado, la información con respecto a la cantidad e intensidad del ejercicio que es necesario para influir positivamente en la cognición es muy escasa. Analizar qué grado de intensidad (baja/alta) como predictor de una mejor o peor repuesta cognitiva es el más adecuado, ayudaría a la integración de este tipo de actividades para su desarrollo en los centros educativos.

Estudios previos han demostrado como con una simple sesión se observan beneficios sobre las funciones ejecutivas (Best, 2011). Por otro lado, otros investigadores han mostrado que tras 6 semanas de empleo de Nintendo Wii Fit en población adulta, no encontraron ningún beneficio tras la intervención. Además, la mayor parte de investigaciones relacionadas con este tema están focalizadas a sectores diferentes al que nos encontramos analizando. Las poblaciones de mayor edad (Zeng, Pope, Lee \& Gao, 2016; Monteiro-Junior, Vaghetii, Nascimiento, Laks \& Deslandes, 2016) 0 poblaciones con algún tipo de enfermedad (De Giglio, Prosperini, Borriello, Bianchi, Pantano Y Pozzilli, 2015; Benzing \& Schmidt, 2017) son las más beneficiadas de las intervenciones realizadas con estos dispositivos.

\section{CONCLUSIÓN}

En conclusión, el uso de la Nintendo Wii presenta un gran potencial para el desarrollo de actividades durante la jornada escolar. La actividad física que se necesita durante el desarrollo de este video juego favorece a una mejora de la cognición en los niños y adolescentes. Por lo tanto, la inclusión de este tipo de actividades en el marco educativo ayudaría a una mayor motivación por parte del alumnado, repercutiendo positivamente en su rendimiento académico. Además, destaca su factor social, beneficiando las relaciones entre iguales y profesorado, así como los beneficios asociados a la salud que presenta la práctica de AF.

\section{REFERENCIAS}

Ardoy, D. N., Fernández-Rodríguez, J. M., Jiménez-Pavón, D., Castillo, R., Ruiz, J. R., \& Ortega, F. B. (2014). A physical education trial improves adolescents' cognitive performance and academic achievement: the EDUFIT study. Scandinavian Journal of Medicine \& Science in Sports, 24(1), e52-61. http://doi.org/10.1111/sms.12093

Benzing, V., \& Schmidt, M. (2017). Cognitively and physically demanding exergaming to improve executive functions of children with attention deficit hyperactivity disorder: a randomised clinical trial. BMC pediatrics, 17(1), 8.

Best, J. R. (2012). Exergaming immediately enhances children's executive function. Developmental psychology, 48(5), 1501. 


\section{EMPLEO DE LA NINTENDO WII PARA LA MEJORA DEL RENDIMIENTO ACADÉMICO}

Chaddock Heyman, L., Hillman, C. H., Cohen, N. J., \& Kramer, A. F. (2014). III. The importance of physical activity and aerobic fitness for cognitive control and memory in children. Monographs of the Society for Research in Child Development, 79(4), 25-50.

De Giglio, L., De Luca, F., Prosperini, L., Borriello, G., Bianchi, V., Pantano, P., \& Pozzilli, C. (2015). A low-cost cognitive rehabilitation with a commercial video game improves sustained attention and executive functions in multiple sclerosis: a pilot study. Neurorehabilitation and neural repair, 29(5), 453-461.

Dutta, N., \& Pereira, M. A. (2015). Effects of Active Video Games on Energy Expenditure in Adults: A Systematic Literature Review. Journal of Physical Activity and Health, 12(6), 890-899.

Flynn, R. M., Richert, R. A., Staiano, A. E., Wartella, E., \& Calvert, S. L. (2014). Effects of exergame play on EF in children and adolescents at a summer camp for low income youth. Journal of educational and developmental psychology, 4(1), 209.

Gao, Z., Chen, S., Pasco, D., \& Pope, Z. (2015). A meta analysis of active video games on health outcomes among children and adolescents. Obesity reviews, 16(9), 783-794.

Gao, Z., Lee, J. E., Pope, Z., \& Zhang, D. (2016). Effect of Active Videogames on Underserved Children's Classroom Behaviors, Effort, and Fitness. Games for Health Journal, 5(5), 318-324.

Haapala, E. A. (2013). Cardiorespiratory fitness and motor skills in relation to cognition and academic performance in children-a review. Journal of human kinetics, 36(1), 55-68.

Joronen, K., Aikasalo, A., \& Suvitie, A. (2016). Nonphysical effects of exergames on child and adolescent well-being: a comprehensive systematic review. Scandinavian Journal of Caring Sciences. http://doi.org/10.1111/scs.12393

LeBlanc, A. G., \& Chaput, J. P. (2016). Pokémon Go: A game changer for the physical inactivity crisis? Preventive Medicine. http://doi.org/10.1016/i.ypmed.2016.11.012

Monteiro-Junior, R. S., Vaghetti, C. A. 0., Nascimento, O. J. M., Laks, J., \& Deslandes, A. C. (2016). Exergames: neuroplastic hypothesis about cognitive improvement and biological effects on physical function of institutionalized older persons. Neural regeneration research, 11(2), 201.

Norris, E., Hamer, M., \& Stamatakis, E. (2016). Active Video Games in Schools and Effects on Physical Activity and Health: A Systematic Review. The Journal of Pediatrics. http://doi.org/10.1016/i.jpeds.2016.02.001

Nurkkala, V. M., Kalermo, J., \& Jarvilehto, T. (2014). Development of exergaming simulator for gym training, exercise testing and rehabilitation. Journal of Communication and Computer, 11, 403411.

Organización Mundial de la Salud (2015). Extraído el 02 de enero de 2016 en http://www.who.int/topics/physical_activity/es/

Ruiz-Ariza, A., Grao-Cruces, A., Loureiro, N. E. M., \& Martínez-López, E. J. (2017a). Influence of physical fitness on cognitive and academic performance in adolescents: A systematic review from 2005-2015. International Review of Sport and Exercise Psychology, 10(1), 108-133. http://doi.org/10.1080/1750984X.2016.1184699

Staiano, A. E., Abraham, A. A., \& Calvert, S. L. (2012). Competitive versus cooperative exergame play for African American adolescents' executive function skills: short-term effects in a longterm training intervention. Developmental psychology, 48(2), 337.

Zeng, N., Pope, Z., Lee, J. E., \& Gao, Z. (2016). A systematic review of active video games on rehabilitative outcomes among older patients. Journal of Sport and Health Science. 